\title{
How to perform aggregometry and lumi- aggregometry in mouse platelets
}

Article

Accepted Version

Hughes, C. E. (2018) How to perform aggregometry and lumiaggregometry in mouse platelets. Platelets, 29 (7). pp. 638643. ISSN 0953-7104 doi:

https://doi.org/10.1080/09537104.2018.1478074 Available at https://centaur.reading.ac.uk/77089/

It is advisable to refer to the publisher's version if you intend to cite from the work. See Guidance on citing.

To link to this article DOI: http://dx.doi.org/10.1080/09537104.2018.1478074

Publisher: Taylor \& Francis

All outputs in CentAUR are protected by Intellectual Property Rights law, including copyright law. Copyright and IPR is retained by the creators or other copyright holders. Terms and conditions for use of this material are defined in the End User Agreement.

\section{www.reading.ac.uk/centaur}

\section{CentAUR}

Central Archive at the University of Reading

Reading's research outputs online 


\title{
How to perform aggregometry and lumi-aggregometry in mouse platelets
}

\author{
Craig E. Hughes \\ Institute for Cardiovascular and Metabolic Research, School of Biological Sciences, University \\ of Reading, Reading, RG6 6AS, UK.
}

Running title: Performing aggregometry in mouse platelets

Author for correspondence: Dr Craig E Hughes, Institute for Cardiovascular and Metabolic Research, School of Biological Sciences, University of Reading, Reading, RG6 6AS, UK, c.e.hughes@reading.ac.uk.

Key words: mouse, platelet, PRP, aggregometry, ADP, PAR4, Optimul

\begin{abstract}
Light transmission aggregometry (LTA) and lumi-aggregometry are the gold standard platelet assays both clinically and for basic research. The availability of different strains of genetically modified mice, and mouse models of human disease means that often laboratories need to use mouse platelets in these assays. Overall, performing aggregometry and lumiaggregometry with mouse platelets is similar to with human platelets, although methods need be adapted to accommodate their small size, reduced blood volume and different protein levels. This review aims to highlight these key considerations when planning aggregometry experiments with mouse platelets. These include the method of taking blood, including the use of anticoagulants, as well as the method of platelet preparation, and how to maximise yields. This review also covers how to maximise the number of aggregations that can be performed, both by understanding the minimum requirements of your aggregometer, or by considering new approaches. These include employing high throughput plate-based aggregometry (Optimul), or the use of TPO-mimetics to stimulate platelet production in mice to boost their platelet counts. Finally, phenotypic differences between mouse and human platelets, such as protein expression or sensitivity to agonists is discussed as an important consideration when planning experiments.
\end{abstract}

\section{INTRODUCTION}

Light transmission aggregometry (LTA) and lumi-aggregometry have remained the gold standard platelet function tests for both clinical diagnostics and in basic research. LTA is a relatively simple assay that measures light transmittance of a stirred platelet suspension which becomes less turbid as the platelets aggregate. Lumi-aggregometry utilises the same basic setup but with the additional capacity to measure ATP secretion from platelet dense granules by the means of luminescence emitted by a luciferin/luciferase reagent added prior to platelet stimulation (Figure 1). These tests have also been widely utilised to characterise the function of mouse platelets from genetically modified mice and for this reason have played as much of a role in our understanding of platelet biology as LTA performed using human platelets.

Several additional challenges face a researcher that wishes to perform experiments on mouse platelets rather than platelets from a human donor. Whereas obtaining mouse platelets requires investment both financially and in the time required to maintain mouse colonies, human blood is relatively easy to obtain once appropriate ethical approval and informed consent has been obtained. In a clinical setting, blood samples are taken from patients in a hospital/clinic, and may then be used for research in a laboratory. In research labs, healthy volunteers may donate blood samples for experiments. Venepuncture is non-invasive and allows volumes of blood ranging from $5-100 \mathrm{~mL}$ to be readily taken without any ill effects on the donor. This provides sufficient amounts for a large number of individual aggregations to be performed. In contrast, only very small volumes of blood (approximately 1-1.2 mL) may be obtained from a mouse. 
The additional challenges of working with murine platelets extend to the more complex considerations during their preparation relative to human platelets. Human platelet preparation for either platelet-rich plasma (PRP) or washed platelets (WP) is simple and straightforward; a slow speed centrifugation step to isolate PRP, followed by higher speed spins to pellet and wash the platelets [1]. Length, speed and numbers of centrifugation, as well as the addition of inhibitors vary by specific purpose and/or the standard protocol in a particular laboratory. Although similar in some regards to the methods utilised to prepare human platelets, due to the smaller volumes of mouse blood samples additional measures must be taken to maximise the volume of PRP or WP obtained from them, both for practical and ethical reasons.

To take advantage of the insights into platelet biology offered by genetically modified mice or mouse models of human disease, methods must be adapted to take into account their small size, reduced blood volume, and different expression levels of proteins. These considerations are the focus of this article, which aims to highlight key considerations when planning aggregometry experiments with mouse platelets, rather than providing a detailed method. For detailed methods, see an excellent methods chapter from Cazenave et al [2].

\section{OBTAINING BLOOD FROM MICE}

Both terminal and non-terminal methods for taking blood from mice can be used. However, taking blood via non-terminal methods, such as via tail vein or saphenous vein, only volumes less than $200 \mu \mathrm{L}$ are permissible within a 24 hour period, assuming the total blood volume for an adult mouse of average size $(25 \mathrm{~g})$ is approximately $1.5 \mathrm{~mL}$. This is based on NC3Rs guidance in the United Kingdom although the value is similar at several universities in the United States. Mice have higher platelet counts than humans, with counts of approximately $1 \times 10^{6} / \mu \mathrm{L}$ often cited, although values ranging from $4.5 \times 10^{5}-1.5 \times 10^{6} / \mu \mathrm{L}$ have been published [3-5]. The reason for this range of published platelet counts may be due to differences between the strain of mice used, but also due to differences in counting method. Most sources, including numerous phenotypic studies on the Mouse Phenome Database (https://phenome.jax.org) agree that the two most commonly used mouse strains, C57BI/6 and Balb/c, both have platelet counts of approximately $1 \times 10^{6} / \mu \mathrm{L}$ [3-7]. Despite having 2-6 fold more platelets per $\mu \mathrm{L}$ than humans, $200 \mu \mathrm{L}$ is unlikely to provide enough platelets for even a single LTA experiment. Furthermore, these methods of blood taking are highly variable with regard to the volume of blood extracted, making accurate matching of anticoagulant volume difficult. Therefore, for LTA experiments, terminal bleeding is the only viable option, excepting retro-orbital bleeding (see below).

Bleeding via cardiac puncture or via the inferior vena cava (in the abdominal cavity) are two commonly used methods of performing a terminal bleed. These can be performed following anaesthesia achieved using either inhalation anaesthetics e.g. isoflurane/ $\mathrm{O}_{2}(5 \%)$, or injected anaesthetics e.g. a mixture of ketamine $(0.2 \%)$ and xylazine (1\%). Alternatively, blood can be taken from a $\mathrm{CO}_{2}$ narcosed mouse, ensuring the blood is drawn immediately following narcosis, as the stasis in blood flow may cause some pre-activation of the platelets. Local rules will most likely guide the selection of anaesthetic, but it is crucial that blood is taken prior to any serious trauma, such as cervical dislocation as this will cause internal bleeding and substantial platelet activation.

Following induction of anaesthesia/narcosis, surgery is performed to open the chest or abdomen to access to the heart or inferior vena cava respectively. A small gauge needle (25G) attached to a $1 \mathrm{~mL}$ syringe should be used to draw the blood. The syringe should contain anticoagulant, most commonly $100 \mu \mathrm{L}$ of acid-citrate-dextrose (ACD), although sodium citrate may be preferable if you want to prepare PRP; the reduced $\mathrm{pH}$ from using ACD reduces platelet function $[2,8,9]$. Another alternative anticoagulant in common use is lowmolecular weight heparin, which has the advantage of maintaining plasma calcium levels. Large volumes (1-1.2 mL or more if the mouse is large) are obtainable by these methods, 
although patience is required, as the last $20 \%$ can be slow once the initial blood pressure is lost. The use of a syringe with these methods allows for accurate recording of volumes and anticoagulant ratios. Immediately following blood taking, the blood is transferred to a tube, containing $200 \mu \mathrm{L}$ of Modified Tyrode's buffer or further anticoagulant, depending on the requirements of the experiment.

Cardiac puncture may also be performed without surgery (but under anaesthesia), by inserting the needle through the skin and without any exteriorisation of the heart. This makes the process quicker, but introduces the risk of incorrectly locating the needle, reducing the yield or possibly resulting in a failed bleed. With practice this method can yield similar volumes of blood as the surgical methods.

Another non-surgical method is retro-orbital bleeding. For this method, a glass capillary tube is coated with the anticoagulant of choice and broken in half, shortening the length and providing a sharp edge at one end. Following anaesthesia, the sharp end of the capillary is inserted behind the eye, into the venous sinus. The blood is withdrawn by capillary action and allowed to drip into a tube containing anticoagulant. This method allows for rapid blood taking of large volumes (1-1.2 $\mathrm{mL}$ ), although there is likely to be a small amount of contaminating tissue fluid. Also, without the use of a syringe, the true volume taken can only be estimated at the time, therefore allowing inaccuracies with anticoagulation. In the UK, this method can only be performed as a terminal procedure, due to the substantial trauma caused to the mouse. However in some countries it is permissible for the mouse to recover, and multiple bleeds $(\sim 500 \mu \mathrm{L})$ performed. Under these circumstances it would be possible to perform a small LTA experiment from a mouse, allow it to recover, and then re-test it at a later date. This could be advantageous for some experiments as platelets from a single mouse could be analysed pre- and post-treatment with a drug, or it could be used as a strategy to maximise platelets from mutant mice which are rare (e.g. low Mendelian frequencies).

\section{PREPARING PRP OR WASHED PLATELETS}

As with preparing human PRP or washed platelets (WP), there are numerous small variations in the methods used for mouse platelets, which won't be detailed here. However, generally speaking the protocol follows the same format as for human platelets, with the major difference being that the volumes are much smaller. As with human platelets, a slow centrifugation step separates PRP from the red cell fraction, from which WP can be isolated with additional higher speed centrifugations.

The PRP prepared from blood drawn from a single mouse is likely to be sufficient for only 2-3 aggregation experiments. However a potential compromise, owing to the greater platelet count of mice, is to dilute the PRP to increase volume without compromising the ability of the sample to aggregate. This can be achieved by performing a platelet count on the isolated PRP and adjusting to a predetermined count by diluting in Modified Tyrode's buffer (counts of between $2 \times 10^{8} / \mathrm{mL}$ to $4 \times 10^{8} / \mathrm{mL}$ are frequently used for aggregometry). This can be supplemented with calcium and fibrinogen to replicate conditions of undiluted plasma, although this may only be needed for specific experiments. As mentioned above, it may be advisable to use either sodium citrate or low-molecular weight heparin as an anticoagulant rather than ACD in this case, as platelet function is compromised if the $\mathrm{pH}$ is below physiological levels [2, 8, 9].

As washing protocols result in some degree of desensitisation of the platelet purinergic receptors; $\mathrm{P}_{2} \mathrm{Y}_{1}$ and $\mathrm{P}_{2} \mathrm{Y}_{12}$ during centrifugation steps, another major advantage of diluting mouse PRP is that the platelets maintain their sensitivity to ADP [10, 11]. However, the presence of plasma proteins may not be compatible with some experiments, for example where inhibitors or agonists are substantially plasma bound. Protocols for washing human platelets which maintain their ADP-sensitivity exist, although at the time of writing, it does not appear that these have been applied to mouse platelets in the literature. These protocols 
usually involve pelleting the platelets with slower speed centrifugations, and in some cases also include apyrase to hydrolyse any ADP present. Potentially these protocols could be applied to mouse platelets, but as mouse platelets are approximately half the size of human platelets ( $4.3 \mathrm{fL}$ compared to $8.4 \mathrm{fL}$ ), they may not pellet well with slower speed centrifugations. Gel filtration is another option to avoid ADP-desensitisation, although it often results in a large dilution of the platelets [12-14]. Therefore, for LTA experiments with mouse platelets, the likelihood is that an additional centrifugation to concentrate the platelets would be required following gel filtration, therefore making the process redundant.

Another difficulty with performing LTA with mouse PRP is the corresponding blank, or Platelet Poor Plasma (PPP) required by the aggregometer. After removing the PRP, the remaining blood can be centrifuged at high speed to obtain PPP although this is most likely to be an insufficient amount for many aggregometers. Therefore dilution with buffer, or to use buffer alone should be considered.

Finally, treatment of the buffy coat when preparing mouse platelets requires consideration. When preparing human platelets, the slower speed centrifugation separates the PRP from the erythrocytes, with the buffy coat easily distinguishable at the interface between the fractions (Figure 2). The buffy coat is avoided when aspirating the PRP as it contains leukocytes. When preparing mouse platelets the buffy coat is often poorly defined following the slow speed centrifugation (Figure 2). Coupled with the significantly smaller volume available compared to a human preparation, means that it is advisable to carefully aspirate as much of the PRP as possible, including the buffy coat. An additional $200 \mu \mathrm{L}$ of Modified Tyrode's buffer can then be added to the remaining red cell fraction, followed by mixing and repeating the slow speed centrifugation. This enables more of the platelets to be separated from the red cell fraction and be combined with the previously aspirated PRP. This step can be repeated as required to increase platelet yield, although with diminishing returns. At this point the PRP from a number of mice can be pooled to increase the amount of platelets for experimentation.

\section{PERFORMING AGGREGATIONS}

Once the PRP or WP are prepared, performing aggregations is largely identical to doing so with human platelets. Two considerations are maximising the number of aggregations that can be performed with the available platelets and the choice of agonists used.

Maximising aggregations - the simplest way to maximise the number of aggregations possible with the amount of platelets available, is to use the lowest platelet count possible. Platelet concentrations of $2 \times 10^{8} / \mathrm{mL}$ are commonly used, although $1.5 \times 10^{8} / \mathrm{mL}$ will also work $[15,16]$. Reducing the platelet concentration lower than this is not advisable, as aggregation becomes less reliable as the platelet concentration is lowered. Minimising the volume used also increases the number of possible aggregations, however, the minimum usable volume will depend on the aggregometer being used due to the position of the light path used to detect changes in light transmittance. Rubber spacers that attach to the bottom of cuvettes to raise the bottom of the cuvette closer to the light path can be used to reduce the minimum volume required. Care should be taken to ensure that the spacers used do not compromise magnetic stirring or light transmission. Furthermore, it is also advisable to confirm that the pipette tips used are of sufficient length to dispense agonist directly into the smaller volume of platelet suspension. Gel loading tips are useful for this purpose. Additionally, some aggregometers have adaptors which allow smaller diameter cuvettes to be used, such as the PAP4 (Bio/Data Corporation).

Recently, an alternative LTA technique that utilises 96-well microtitre plates rather than glass cuvettes has become widely utilised to measure aggregation of human platelets. Optical multichannel (Optimul) aggregometry is a high throughput adaptation of LTA that measures aggregation at an endpoint rather in real time and utilises an orbital plate shaker instead of stir bars [17-19]. Use of half-area 96-well plates reduces the volume of PRP or WP required to 40 
$\mu \mathrm{L}$ per aggregation. The end-point nature of the assay means that all 96 aggregations can be performed simultaneously and measured using a plate reader with the capability to read absorbance (Figure 1). Currently this method has not been adapted to enable measurement of ATP secretion via luminescence; therefore it is not compatible for studying platelet dense granule secretion.

Recently the Optimul method has been utilised to measure aggregation of mouse platelets [20]. The authors of this study reported that that, unlike human, mouse platelets do not aggregate well to some standard platelet agonists using this method. Using Optimul, mouse platelets do not respond to arachidonic acid, ristocetin, adrenaline or U46619. Mouse platelets do respond to ADP, as well as to collagen, although unlike human platelets, not to concentrations below $5 \mu \mathrm{g} / \mathrm{mL}$. Mouse platelets also respond to a PAR4 activating peptide in this assay. We have also performed similar experiments and our data agrees, with no substantial aggregation seen with arachidonic acid and the most robust aggregation seen with a PAR4 peptide (Figure 3). Therefore, depending on the experiment, Optimul may be a viable option for maximising the amount of aggregations possible with mouse platelets.

In the case of mutant mice which are thrombocytopenic, these strategies may still yield too few platelets for LTA experiments. In these instances, an option is to increase the platelet count in the mice prior to experimentation. Thrombocytopenia in humans can be treated clinically with either the TPO-analogue romiplostim (trade name Nplate, produced by Amgen), or the small molecule TPO receptor agonist eltrombopag (trade names Revolade (EU) and Promacta (USA), produced by GlaxoSmithKline). A study from 2012 used romiplostim to rescue the thrombocytopenia in $\mathrm{Myh}^{-/}$mice [21]. Mice were dosed with $100 \mu \mathrm{g} / \mathrm{kg}$ romiplostim every 3 days, boosting their platelet levels by 2.5-fold [21]. Interestingly, the platelet counts of the wild type control mice were boosted by 8-fold over a four week dosing regimen, with maximal platelet counts of approximately $6.5 \times 10^{6} / \mu \mathrm{L}$ being reported. In a study from 2017, the response to romiplostim was compared between adult and neonatal mice [22]. Mice were given a single dose of romiplostim $(10-300 \mu \mathrm{g} / \mathrm{kg})$ and platelet counts were measured daily for 14 days. Platelet counts peaked between 5-7 days, but were boosted to a lesser extent, increasing by a maximum of 4.2-fold [22]. A third study, from 2015 used romiplostim to recover platelet counts in mice following chemotherapy-induced thrombocytopenia [23]. In this case, mice were dosed within 3 days of chemotherapy-induced thrombocytopenia, with a range of concentrations of romiplostim $(10-1000 \mu \mathrm{g} / \mathrm{kg})$. The researchers found that does of $100 \mu \mathrm{g} / \mathrm{kg}$ and above were able to reduce the level thrombocytopenia and increase the platelet recovery time, however they did not measure the effect of romiplostim on untreated mice [23]. These studies show the potential of romiplostim to boost platelet counts in mice such that the blood from a single mouse could yield similar numbers of platelets as $20-30 \mathrm{~mL}$ of human blood. However, it should be noted that the use of romiplostim also caused platelets to be larger and less mature [21, 22]. Therefore, experiments using romiplostim to boost platelet counts would require important controls to confirm that platelets from romiplostim-treated mice are not phenotypically different to those from untreated mice.

Agonist selection - The final consideration when designing an aggregation experiment with mouse platelets is the choice of agonist. In general, regarding agonist choice, an experimental design for human platelets can also be used for mouse platelets, with two notable exceptions. Mouse platelets do not express the thrombin receptor PAR1. Therefore, the PAR1 activating peptide, TRAP6 cannot be used for LTA with mouse platelets. Alternatively the PAR4 activating peptide should be used for LTA with either PRP or WP, or thrombin can be used, but for WP only as thrombin will cleave the fibrinogen present in plasma forming a clot [24]. Mouse platelets also do not express the low affinity lgG receptor FcyRIIA, in fact the gene is absent from the mouse genome, only being found in primates. FcyRIIA is activated by the Fc portion of IgG antibodies, and also by the specific antibody IV.3. FcyRIIA signalling occurs both following ligand binding and in a cooperative manner with the integrin allb $\beta 3$ [25-27], and 
it is the mechanism of platelet activation in Heparin-Induced Thrombocytopenia (HIT) [28]. The absence of FcyRIIA from mouse makes the use of other activatory antibodies easier with mouse platelets, as there is no non-specific binding to FcyRIIA to take into consideration; in human platelets, FcyRIIA must be blocked with IV.3 F(ab) fragments when using antibodies as agonists to prevent platelet activation through the $\lg G$ receptor.

For agonists which activate both mouse and human platelets, there are small differences in sensitivity which may need to be taken into consideration depending on the aim of the experiment. For example, it has been shown that mouse platelets are more sensitive to both ADP and thrombin than human platelets, although it should be noted that this was found by measuring fibrinogen binding rather than by aggregometry [29]. Another example is aggregation stimulated by the podoplanin receptor CLEC-2. Proteomic data suggests that mouse platelets express approximately 10 -fold more CLEC-2 compared to human platelets, resulting in a slight increase in sensitivity to CLEC-2 agonists [30, 31]. However, the most obvious manifestation of this difference is seen with the dependence of human platelets on secondary mediators; apyrase and indomethacin inhibit CLEC-2 mediated aggregation in humans, but not in mouse platelets $[32,33]$. Differences in expression of receptors between species should therefore be considered when designing experiments.

\section{CONCLUSION}

Overall, performing aggregometry and lumi-aggregometry with mouse platelets is similar to with human platelets. However, important additional considerations must be taken into account when planning experiments such as the method of taking blood, use of anticoagulants and the method of platelet preparation, to maximise yield. Understanding the minimum volume requirements of an aggregometer will maximise the number of aggregations that can be performed, and this can be taken further by employing high throughput plate-based aggregometry (Optimul). Finally, some agonists are not appropriate for mouse platelets, whereas others may show subtle differences in sensitivity between species.

\section{ACKNOWLEDGMENTS}

The author would like to thank Dr Alice Pollitt and Dr Alex Bye for critical reading of the manuscript, and Dr Marie Lordkipanidze for performing Optimul experiments.

\section{DECLARATION OF INTERESTS}

The author has no conflicts of interest to declare.

\section{REFERENCES}

1 Mustard JF, Perry DW, Ardlie NG, Packham MA. Preparation of suspensions of washed platelets from humans. British journal of haematology. 1972; 22: 193-204.

2 Cazenave JP, Ohlmann P, Cassel D, Eckly A, Hechler B, Gachet C. Preparation of washed platelet suspensions from human and rodent blood. Methods Mol Biol. 2004; 272: 13-28. 10.1385/1-59259-782-3:013.

3 Levin J, Ebbe S. Why are recently published platelet counts in normal mice so low? Blood. 1994; 83: 3829-31.

4 Longmore GD, Pharr P, Neumann D, Lodish HF. Both megakaryocytopoiesis and erythropoiesis are induced in mice infected with a retrovirus expressing an oncogenic erythropoietin receptor. Blood. 1993; 82: 2386-95.

5 Harrison J, Kappas A, Levere RD, Lutton JD, Chertkov JL, Jiang S, Abraham NG. Additive effect of erythropoietin and heme on murine hematopoietic recovery after azidothymidine treatment. Blood. 1993; 82: 3574-9.

6 Fukuda T, Asou E, Nogi K, Goto K. Evaluation of mouse red blood cell and platelet counting with an automated hematology analyzer. J Vet Med Sci. 2017; 79: 1707-11. 10.1292/jvms.17-0387. 
7 Barrios M, Rodriguez-Acosta A, Gil A, Salazar AM, Taylor P, Sanchez EE, Arocha-Pinango $\mathrm{CL}$, Guerrero B. Comparative hemostatic parameters in BALB/C, C57BL/6 and C3H/He mice. Thrombosis research. 2009; 124: 338-43. 10.1016/j.thromres.2008.11.001.

8 Marumo M, Suehiro A, Kakishita E, Groschner K, Wakabayashi I. Extracellular pH affects platelet aggregation associated with modulation of store-operated $\mathrm{Ca}(2+)$ entry. Thrombosis research. 2001; 104: 353-60.

9 Aster RH, Jandl JH. Platelet Sequestration in Man. I. Methods. The Journal of clinical investigation. 1964; 43: 843-55. 10.1172/JCl104970.

10 Ardlie NG, Perry DW, Packham MA, Mustard JF. Influence of apyrase on stability of suspensions of washed rabbit platelets. Proc Soc Exp Biol Med. 1971; 136: 1021-3.

11 Baurand A, Eckly A, Bari N, Leon C, Hechler B, Cazenave JP, Gachet C. Desensitization of the platelet aggregation response to ADP: differential down-regulation of the P2Y1 and P2cyc receptors. Thrombosis and haemostasis. 2000; 84: 484-91.

12 Lages B, Scrutton MC, Holmsen $\mathrm{H}$. Studies on gel-filtered human platelets: isolation and characterization in a medium containing no added $\mathrm{Ca} 2+, \mathrm{Mg} 2+$, or $\mathrm{K}+$. J Lab Clin Med. 1975; 85: 811-25.

13 Tangen O, Berman HJ. Gel filtration of blood platelets: a methodological report. Advances in experimental medicine and biology. 1972; 34: 235-43.

14 Tangen O, McKinnon EL, Berman HJ. On the fine structure and aggregation requirements of gel filtered platelets (GFP). Scand J Haematol. 1973; 10: 96-105.

15 Nanda N, Bao M, Lin H, Clauser K, Komuves L, Quertermous T, Conley PB, Phillips DR, Hart MJ. Platelet endothelial aggregation receptor 1 (PEAR1), a novel epidermal growth factor repeat-containing transmembrane receptor, participates in platelet contact-induced activation. The Journal of biological chemistry. 2005; 280: 24680-9. 10.1074/jbc.M413411200.

16 Popp M, Thielmann I, Nieswandt B, Stegner D. Normal Platelet Integrin Function in Mice Lacking Hydrogen Peroxide-Induced Clone-5 (Hic-5). PloS one. 2015; 10: e0133429. 10.1371/journal.pone.0133429.

17 Lordkipanidze M, Lowe GC, Kirkby NS, Chan MV, Lundberg MH, Morgan NV, Bem D, Nisar SP, Leo VC, Jones ML, Mundell SJ, Daly ME, Mumford AD, Warner TD, Watson SP, Genotyping UK, Phenotyping of Platelets Study G. Characterization of multiple platelet activation pathways in patients with bleeding as a high-throughput screening option: use of 96-well Optimul assay. Blood. 2014; 123: e11-22. 10.1182/blood-2013-08-520387.

18 Bye AP, Unsworth AJ, Desborough MJ, Hildyard CAT, Appleby N, Bruce D, Kriek N, Nock $\mathrm{SH}$, Sage T, Hughes CE, Gibbins JM. Severe platelet dysfunction in NHL patients receiving ibrutinib is absent in patients receiving acalabrutinib. Blood Adv. 2017; 1: 2610-23. 10.1182/bloodadvances.2017011999.

19 Chan MV, Armstrong PC, Papalia F, Kirkby NS, Warner TD. Optical multichannel (optimul) platelet aggregometry in 96-well plates as an additional method of platelet reactivity testing. Platelets. 2011; 22: 485-94. 10.3109/09537104.2011.592958.

20 Smith CW, Thomas SG, Raslan Z, Patel P, Byrne M, Lordkipanidze M, Bem D, Meyaard L, Senis YA, Watson SP, Mazharian A. Mice Lacking the Inhibitory Collagen Receptor LAIR-1 Exhibit a Mild Thrombocytosis and Hyperactive Platelets. Arteriosclerosis, thrombosis, and vascular biology. 2017; 37: 823-35. 10.1161/ATVBAHA.117.309253.

21 Leon C, Evert K, Dombrowski F, Pertuy F, Eckly A, Laeuffer P, Gachet C, Greinacher A. Romiplostim administration shows reduced megakaryocyte response-capacity and increased myelofibrosis in a mouse model of MYH9-RD. Blood. 2012; 119: 3333-41. 10.1182/blood2011-08-373811.

22 Sparger KA, Ramsey H, Lorenz V, Liu ZJ, Feldman HA, Li N, Laforest T, Sola-Visner MC. Developmental differences between newborn and adult mice in response to romiplostim. Platelets. 2017: 1-8. 10.1080/09537104.2017.1316481.

23 McElroy PL, Wei P, Buck K, Sinclair AM, Eschenberg M, Sasu B, Molineux G. Romiplostim promotes platelet recovery in a mouse model of multicycle chemotherapy-induced thrombocytopenia. Experimental hematology. 2015; 43: 479-87. 10.1016/j.exphem.2015.02.004. 
24 Michelson AD. Platelet activation by thrombin can be directly measured in whole blood through the use of the peptide GPRP and flow cytometry: methods and clinical applications. Blood Coagul Fibrinolysis. 1994; 5: 121-31.

25 Boylan B, Gao C, Rathore V, Gill JC, Newman DK, Newman PJ. Identification of FcgammaRIla as the ITAM-bearing receptor mediating alphallbbeta3 outside-in integrin signaling in human platelets. Blood. 2008; 112: 2780-6. 10.1182/blood-2008-02-142125.

26 Gao C, Boylan B, Bougie D, Gill JC, Birenbaum J, Newman DK, Aster RH, Newman PJ. Eptifibatide-induced thrombocytopenia and thrombosis in humans require FcgammaRIla and the integrin beta3 cytoplasmic domain. The Journal of clinical investigation. 2009; 119: 50411. $10.1172 / \mathrm{JCl} 36745$.

27 Hughes CE, Finney BA, Koentgen F, Lowe KL, Watson SP. The N-terminal SH2 domain of Syk is required for (hem)ITAM, but not integrin, signaling in mouse platelets. Blood. 2015; 125: 144-54. 10.1182/blood-2014-05-579375.

28 Reilly MP, Taylor SM, Hartman NK, Arepally GM, Sachais BS, Cines DB, Poncz M, McKenzie SE. Heparin-induced thrombocytopenia/thrombosis in a transgenic mouse model requires human platelet factor 4 and platelet activation through FcgammaRIIA. Blood. 2001; 98: 2442-7.

29 Nylander S, Mattsson C, Lindahl TL. Characterisation of species differences in the platelet ADP and thrombin response. Thrombosis research. 2006; 117: 543-9. 10.1016/j.thromres.2005.04.026.

30 Burkhart JM, Vaudel M, Gambaryan S, Radau S, Walter U, Martens L, Geiger J, Sickmann A, Zahedi RP. The first comprehensive and quantitative analysis of human platelet protein composition allows the comparative analysis of structural and functional pathways. Blood. 2012; 120: e73-82. 10.1182/blood-2012-04-416594.

31 Zeiler M, Moser M, Mann M. Copy number analysis of the murine platelet proteome spanning the complete abundance range. Mol Cell Proteomics. 2014; 13: 3435-45. 10.1074/mcp.M114.038513.

32 Pollitt AY, Grygielska B, Leblond B, Desire L, Eble JA, Watson SP. Phosphorylation of CLEC-2 is dependent on lipid rafts, actin polymerization, secondary mediators, and Rac. Blood. 2010; 115: 2938-46. 10.1182/blood-2009-12-257212.

33 Borgognone A, Navarro-Nunez L, Correia JN, Pollitt AY, Thomas SG, Eble JA, Pulcinelli FM, Madhani M, Watson SP. CLEC-2-dependent activation of mouse platelets is weakly inhibited by cAMP but not by cGMP. J Thromb Haemost. 2014; 12: 550-9. 10.1111/jth.12514.

\section{FIGURES}

FIGURE 1 - Schematic of light transmission lumi-aggregometry and Optimul. A) Light Transmission Aggregometry (LTA): A sample of PRP is turbid, allowing only a proportion of the incident light to be transmitted. Following agonist induced platelet aggregation the sample is less turbid, allowing more light to be transmitted. If performing lumi-aggregometry, light is also emit from the enzyme luciferase, following ATP secretion from platelet dense granules. B) Optical multichannel (Optimul) aggregometry: Multiple wells of a 96-well microtitre plate are filled with PRP for simultaneous aggregations. Following agonist induced platelet aggregation the plate is read in a plate reader, and the endpoint light transmission is calculated.

FIGURE 2 - Comparison of human and mouse platelet preparations. Following the first slow speed centrifugations, human blood is separated into three clearly defined layers, with the buffy coat separating the Platelet-rich plasma (PRP) and the erythrocyte layers. With mouse blood, the buffy coat layer is less clearly defined. When aspirating human PRP, the buffy coat is avoided, but when aspirating mouse PRP the buffy coat should also be removed to maximise platelet yield.

FIGURE 3 - Optical multichannel (Optimul) aggregometry with mouse platelets. 96-well microtitre plates were coated with gelatine to block surface activation of platelets prior to addition of the platelet agonists ( $\mathrm{AA}=$ arachidonic acid). $40 \mu \mathrm{L}$ of PRP (or PPP as a control) 
was added into the wells and the plate was placed on an orbital plate shaker, heated at $37^{\circ} \mathrm{C}$ and mixed at $1200 \mathrm{rpm}$, for 5 minutes. Absorbance was then measured at $650 \mathrm{~nm}$ on a 96well plate reader. Platelet aggregation was expressed as the maximal percent change in light transmittance from PRP wells in response to agonists, using PPP as reference, $n=5-7$, error bars represent the standard error of the mean (s.e.m.). 